\title{
Dissipativity and Stabilization of Nonlinear Repetitive Processes ${ }^{\text {th }}$
}

\author{
Pavel Pakshin ${ }^{\mathrm{a}, *}$, Julia Emelianova, Mikhail Emelianov ${ }^{\mathrm{b}}$, Krzysztof \\ Gałkowskic $^{\mathrm{c}}$, Eric Rogers ${ }^{\mathrm{d}}$ \\ ${ }^{a}$ Arzamas Polytechnic Institute of R.E. Alekseev Nizhny Novgorod State Technical \\ University ,19, Kalinina Street, Arzamas, 607227, Russia and Lobachevsky State \\ University of Nizhny Novgorod, Prospekt Gagarina, 23, 603950, Nizhny Novgorod, Russia \\ ${ }^{b}$ Arzamas Polytechnic Institute of R.E. Alekseev Nizhny Novgorod State Technical \\ University ,19, Kalinina Street, Arzamas, 607227, Russia \\ ${ }^{c}$ Institute of Control and Computation Engineering, University of Zielona Góra, \\ ul. Podgórna 50, 65-246 Zielona Góra, Poland. \\ ${ }^{d}$ Department of Electronics and Computer Science, University of Southampton, \\ Southampton SO17 1BJ, UK.
}

\begin{abstract}
Repetitive processes are characterized by repeated executions of a task defined over a finite duration with resetting after each execution is complete. Also the output from any execution directly influences the output produced on the next execution. The repetitive process model structure arises in the modeling of physical processes and can also be used to effect in the control of other systems, such as iterative learning control where experimental verification of designs has been reported. The existing systems theory for them is, in the main, linear model based. This paper considers nonlinear repetitive processes using a dissipative setting and develops a stabilizing control law with the required conditions expressed in terms of vector storage functions. This design is then extended to stabilization plus disturbance attenuation.
\end{abstract}

Keywords: nonlinear repetitive processes, stability, dissipativity, passivity,

\footnotetext{
«his work is performed in framework of contract No 2.1748.2014/K with Ministry of Education and Science of Russia and also supported in part by the Russian Foundation for Basic Research under grant $13-08-01092 \_a$; Sec.3 is supported solely by Russian Science foundation under grant RSF 14-29-00142

* Corresponding author.

Email address: pakshin@apingtu.edu.ru (Pavel Pakshin)
} 
$\mathcal{H}_{\infty}$ disturbance attenuation.

\section{Introduction}

The systems considered in this paper make a series of sweeps, also termed passes, through dynamics defined over a fixed finite duration termed the pass length [1]. On completion of each pass, the system resets to the starting location and the next pass can begin, either immediately after the resetting is complete or after a period of time has elapsed. Each pass output is termed the pass profile and the distinguishing feature of these systems is that the pass profile on the previous pass acts as a forcing function on, and hence contributes to, the dynamics of the next pass profile. Such systems are known as repetitive processes.

Repetitive processes propagate information over a subset of the upperright quadrant of the $2 \mathrm{D}$ plane and in this paper the notation for variables is of the form $y_{k}(t)$ where $y$ is the scalar or vector valued variable, the nonnegative integer $k$ denotes the pass number and $t \in[0, T]$ denotes the temporal variable defined over the finite duration pass length $T<\infty$, where the dynamics in the temporal variable can be discrete or differential. An industrial example described in [1], with references to the original modeling work, is long-wall coal cutting where the pass profile is the height of the stone/coal interface above some datum line and the objective is to extract the maximum amount of coal without penetrating the stone/coal boundary. The cutting machine rests on the most recently produced pass profile during the production of the next pass profile and therefore this is an industrial repetitive process. The unique control problem is that the sequence of pass profiles $\left\{y_{k}\right\}$ generated can contain oscillations that increase in amplitude from passto-pass, that is, with $k$.

If these oscillations occur in a mining or other industrial example, solving a stabilization problem is the alternative to lost production resulting from a down time to enable their manual removal. This problem cannot, however, be solved using standard, or 1D, systems theory/algorithms since this approach ignores their inherent 2D systems structure, that is, information propagation occurs from pass-to-pass and along a given pass. Also the initial conditions are reset before the start of each new pass.

The available results on the control of linear repetitive processes starts from a stability theory developed [1] using an abstract model of the dynamics 
in a Banach space setting that includes a very large number of processes with linear dynamics and a constant pass length as special cases. The existence of this theory has also led to the emergence of problem areas where using a repetitive process setting for analysis has advantages. An example is classes of Iterative Learning Control (ILC) laws where experimental verification has been reported [2]. Another area is the analysis of OL-Nash games with a gas pipeline application [3].

If a linear model approximation of the dynamics is not possible then comparatively much less work has been reported on the stability of nonlinear 2D (or $n D, n>2$ ) systems, see, e.g., [4, 5] and references therein. The continual emergence of new possible application areas, such as ILC applied to bead morphology in laser metal deposition processes [6], adds further support to the development of this general area, starting from a stability theory and leading on to control law design.

It is important to place repetitive processes in context and, in particular, explain why they cannot be controlled by standard theory. These processes differ from repetitive control, see, e.g., [7], where the reference signal is periodic and there is no stoppage time between the end of one cycle and the start of the next one. Repetitive processes do have a resetting after each pass is complete and moreover the structure of the boundary conditions is critical to the stability and hence control properties.

In the simplest case, the initial state on each pass is independent of the previous pass dynamics but in some applications this assumption is too simplistic and it is necessary to have state initial conditions that are an explicit function of the dynamics produced on the previous pass. Such conditions arise in the long wall coal cutting example, see [1] for a detailed explanation that cites the original work in this area. Examples exist [1] where ignoring the resetting and thereby converting the dynamics into an 'equivalent' infinite pass length standard system gives incorrect conclusions as does modeling the state initial conditions as pass independent when they are actually pass dependent. Pass dependent boundary conditions also arise in the use of repetitive process stability theory to study iterative solution algorithms for nonlinear dynamic optimal control problems based on the maximum principle [8]. Hence there is a need in both theory and applications for a rigorous stability theory for these processes that extends to control law design.

In the case of 1D nonlinear systems, dissipativity theory [9] is one of the most powerful methods for control design, where a particular form, known as passivity (and its generalizations) see, e.g., [10] plays a significant role in 
solving the global feedback stabilization problem for a wide class of systems. This paper gives new results on a dissipativity approach to the stabilization of discrete and differential nonlinear repetitive processes, where the term 'differential' refers to dynamics along the pass governed by a matrix differential equation for which the term continuous could equally be used. The results are obtained using a vector storage function approach that is different from that in $[11,12]$ and results in a control law to guarantee exponential stability of the controlled process. This design is then extended to stabilization plus disturbance attenuation.

\section{Dissipativity and the stabilization of discrete repetitive pro- cesses}

\subsection{Process description and definitions}

The discrete nonlinear repetitive processes considered in this section are described by the following state-space model over $0 \leq p \leq T-1, k \geq 0$,

$$
\begin{aligned}
x_{k+1}(p+1) & =f_{1}\left(x_{k+1}(p), y_{k}(p), u_{k+1}(p)\right), \\
y_{k+1}(p) & =f_{2}\left(x_{k+1}(p), y_{k}(p), u_{k+1}(p)\right),
\end{aligned}
$$

where the integer $T<\infty$ denotes the number of samples over the pass length and on pass $k x_{k}(p) \in \mathbb{R}^{n_{x}}$ is the current pass state vector, $y_{k}(p) \in \mathbb{R}^{n_{y}}$ is the pass profile vector, $f_{1}$, and $f_{2}$ are nonlinear functions such that $f_{1}(0,0,0)=$ $0, f_{2}(0,0,0)=0$. This last requirement is necessary to have zero as the equilibrium state. The boundary conditions, that is, the pass state initial vector sequence and the initial pass profile, are assumed to be of the form

$$
\begin{aligned}
x_{k+1}(0) & =d_{k+1}, \quad k \geq 0, \\
y_{0}(p) & =f(p), 0 \leq p \leq T-1,
\end{aligned}
$$

where the entries in $d_{k+1} \in \mathbb{R}^{n_{x}}$ are known constants and the entries in $f(p) \in \mathbb{R}^{n_{y}}$ are known functions of $p$. It is assumed that $d_{k+1}$ and $f(p)$ have bounded energy, that is, there exist finite real numbers $M_{f}>0, \kappa_{d}>0$ and $0<\lambda_{d}<1$ such that $d_{k+1}$ and $f(p)$ satisfy

$$
|f(p)|^{2} \leq M_{f},\left|d_{k+1}\right|^{2} \leq \kappa_{d} \lambda_{d}^{k+1}, k \geq 0
$$

where $|q|$ denotes the Euclidian norm of a vector $q$. 
The scalar $\left|M_{f}\right|^{\frac{1}{2}}$ is an upper bound on the norm of the initial pass profile and $\kappa_{d}^{\frac{1}{2}}$ is an upper bound on the initial pass state vector sequence, which is assumed to be bounded in norm by an exponentially decreasing sequence with rate of convergence (in $k$ ) $\lambda_{d}$. In particular, $\lambda_{d}$ represents the rate of convergence in $k$ of the pass initial state vector sequence. From this point onwards, all references to the boundary conditions for the processes considered will assume that they satisfy (3). Moreover, in this paper the state initial vector on each pass is independent of the previous pass profile vector but, as discussed in the previous section, there are applications where this assumption is too strong. Sufficient progress with the case considered in this paper should prompt further research on this more general case.

In the control and systems theory developed for linear repetitive processes, the stability along the pass property has formed the basis for control law design and experimental verification $[1,2]$. This property demands that a bounded initial pass profile produces a bounded sequence of pass profiles for all possible values of the pass length and is based on linear operator theory in a Banach space setting. Hence it cannot be directly transferred to the nonlinear case.

Stability along the pass requires that the sequence (in $k$ ) of pass profiles and state vectors are bounded independent of the pass length. In the case of nonlinear discrete nonlinear repetitive processes stability should also enforce boundedness (in $k$ ) independent of the pass length of the sequences (in $k$ ) of pass profiles and state vectors and one possible approach would be to use a Lyapunov function approach as in the stability analysis of $1 \mathrm{D}$ nonlinear systems.

The Lyapunov approach is based on properties of the function itself and for discrete dynamics of its increments, but the dynamics of repetitive processes are determined by the state vector $x$ and the pass profile vector $y$, which are functions of the two independent variables $p$ and $k$. A candidate Lyapunov function for these processes can be chosen as a scalar function, $V(x, y)$, but to construct the gradient along the trajectories of (1) it is required to have $x_{k+1}(p)-x_{k}(p)$ and $y_{k}(p+1)-y_{k}(p)$ as functions of $x$ and $y$. These quantities can only be found by solving (1) but then all of the advantages of the Lyapunov approach are lost.

A powerful method in the analysis and control of 1D systems is dissipativity theory [9], especially the particular case of passivity theory $[9,10]$ where an extension of a Lyapunov function termed a storage function is used. 
Previous work [13, 14] developed a stability theory for discrete nonlinear repetitive processes based on the use of vector Lyapunov functions and the discrete counterpart of their divergence along the trajectories to characterize the property of exponential stability. In this paper the problem considered is stabilization and disturbance attenuation using a pass profile based control law, for discrete dynamics in this section and differential in the next.

Consider a control law of the form

$$
u=\psi(x, y), \psi(0,0)=0 .
$$

Then the following is the definition of exponential stability for the controlled process.

Definition 1. Consider the discrete nonlinear repetitive process resulting from the application of the control law of the form (4) to (1) and (2). Then the resulting controlled process is said to be exponentially stable if there exist real numbers $\kappa>0$ and $0<\lambda<1$ such that

$$
\left|x_{k}(p)\right|^{2}+\left|y_{k}(p)\right|^{2} \leq \kappa \lambda^{k+p} .
$$

This paper builds on this previous work [13, 14] on stability alone to develop a dissipativity theory for discrete dynamics in this section and differential dynamics in the next. The result is a stabilizing control law with an extension to disturbance rejection for discrete dynamics.

The analysis uses a vector storage function of the form

$$
V\left(x_{k+1}(p), y_{k}(p)\right)=\left[\begin{array}{c}
V_{1}\left(x_{k+1}(p)\right) \\
V_{2}\left(y_{k}(p)\right)
\end{array}\right]
$$

where $V_{1}(x)>0, x \neq 0, \quad V_{2}(y)>0, y \neq 0, \quad V_{1}(0)=0, V_{2}(0)=0$. Also the discrete counterpart of the divergence operator of this function along the trajectories of $(1)$ is

$$
\mathcal{D}_{d} V\left(x_{k+1}(p), y_{k}(p)\right)=\Delta_{p} V_{1}\left(x_{k+1}(p)\right)+\Delta_{k} V_{2}\left(y_{k}(p)\right),
$$

where

$$
\begin{aligned}
\Delta_{p} V_{1}\left(x_{k+1}(p)\right) & =V_{1}\left(x_{k+1}(p+1)\right)-V_{1}\left(x_{k+1}(p)\right), \\
\Delta_{k} V_{2}\left(y_{k}(p)\right) & =V_{2}\left(y_{k+1}(p)\right)-V_{2}\left(y_{k}(p)\right) .
\end{aligned}
$$


Introduce, for analysis and control law design purposes only, the auxiliary vector $z_{k}(p) \in \mathbb{R}^{n_{z}}$ given by

$$
z_{k+1}(p)=g\left(x_{k+1}(p), y_{k}(p), u_{k+1}(p)\right)
$$

where $g$ is a nonlinear function such that $g(0,0,0)=0$ and define the dissipativity property as follows.

Definition 2. A discrete nonlinear repetitive process described by (1) and (2) is said to be exponentially dissipative if there exists a vector function (6), a scalar function $S(u, z)$ and positive scalars $c_{1}, c_{2}$ and $c_{3}$ such that

$$
\begin{aligned}
c_{1}|x|^{2} & \leq V_{1}(x) \leq c_{2}|x|^{2}, \\
c_{1}|y|^{2} & \leq V_{2}(y) \leq c_{2}|y|^{2}, \\
\mathcal{D}_{d} V\left(x_{k+1}(p), y_{k}(p)\right) & \leq S\left(u_{k+1}(p), z_{k+1}(p)\right) \\
& -c_{3}\left(\left|x_{k+1}(p)\right|^{2}+\left|y_{k}(p)\right|^{2}\right) .
\end{aligned}
$$

In the particular case when $S(u, z)=z^{T} G u$, where $G$ is a constant matrix of compatible dimensions, a discrete nonlinear repetitive process described by (1) and (2) is said to be exponentially $G$-passive, see [15] for the 1D systems case. Since (1) does not involve full increments, as in the case of ordinary 1D difference equations, it is impossible to use cross terms in the vector storage function (6).

The auxiliary vector $z$ of (8) can be used to achieve certain dissipativity properties and for the case of passivity this is known as passivation [16]. Moreover, the choice of this vector depends on the choice of storage function $V$ and it is a separate complex problem (similar to the choice of a Lyapunov function for a nonlinear system). The problem is to find a pair $(z, V)$ satisfying (11) and, later in this section, it is shown how this pair and a stabilizing control law can be obtained for a particular applications relevant special case.

\subsection{Passivity and stabilization}

The following theorem is the first major new result of this paper.

Theorem 1. Suppose that a discrete nonlinear repetitive process described by (1) and (2) is exponentially $G$ passive. Suppose also that there exists a function $\varphi(z)$ such that $\varphi(0)=0$ and $z^{T} G \varphi(z)>0$ if $z \neq 0$. Then the control law

$$
u_{k+1}(p)=u\left(z_{k+1}(p)\right)=-\varphi\left(z_{k+1}(p)\right)
$$

results in controlled dynamics that are exponentially stable. 
Proof. It follows from (11) that it is possible to choose $\bar{c}_{3}$ small enough such that $\bar{c}_{3}<c_{3}$ and

$$
\begin{aligned}
\mathcal{D}_{d} V\left(x_{k+1}(p), y_{k}(p)\right) & \leq-z_{k+1}^{T}(p) G \varphi\left(z_{k+1}(p)\right)-c_{3}\left(\left|x_{k+1}(p)\right|^{2}+\left|y_{k}(p)\right|^{2}\right) \\
& \leq-\bar{c}_{3}\left(\left|x_{k+1}(p)\right|^{2}+\left|y_{k}(p)\right|^{2}\right) .
\end{aligned}
$$

Using (9), (10) and (11), (13) can be rewritten as

$$
\begin{aligned}
V_{1}\left(x_{k+1}(p+1)\right) & -V_{1}\left(x_{k+1}(p)\right)+V_{2}\left(y_{k+1}(p)\right)-V_{2}\left(y_{k}(p)\right) \\
& \leq-\bar{c}_{3}\left(\left|x_{k+1}(p)\right|^{2}+\left|y_{k}(p)\right|^{2}\right) \\
& \leq-\frac{\bar{c}_{3}}{c_{2}}\left(V _ { 1 } \left(x_{k+1}(p)+V_{2}\left(y_{k}(p)\right),\right.\right.
\end{aligned}
$$

or

$$
V_{1}\left(x_{k+1}(p+1)\right)+V_{2}\left(y_{k+1}(p)\right) \leq\left(1-\frac{\bar{c}_{3}}{c_{2}}\right)\left(V _ { 1 } \left(x_{k+1}(p)+V_{2}\left(y_{k}(p)\right)\right.\right.
$$

Define $\lambda=1-\frac{\bar{c}_{3}}{c_{2}}$ and choose $\bar{c}_{3}$ to satisfy

$$
\lambda_{d}^{\frac{1}{2}}<\lambda<1
$$

where the left-hand side inequality in this expression is necessary to establish convergence of the series at the end of the proof. Rewrite (15) as

$$
\begin{aligned}
V_{1}\left(x_{k+1}(p+1)\right) & \leq \lambda V_{1}\left(x_{k+1}(p)\right)+\lambda V_{2}\left(y_{k}(p)\right) \\
& -V_{2}\left(y_{k+1}(p)\right) .
\end{aligned}
$$

Solving recursively inequality (17) with respect to $V_{1}\left(x_{k+1}(p)\right)$ we obtain an expression in terms of the boundary conditions:

$$
\begin{aligned}
V_{1}\left(x_{k+1}(p)\right) & \leq V_{1}\left(x_{k+1}(0)\right) \lambda^{p} \\
& +\sum_{h=0}^{p-1}\left[\lambda V_{2}\left(y_{k}(h)\right)-V_{2}\left(y_{k+1}(h)\right)\right] \lambda^{p-h-1}
\end{aligned}
$$

and on introducing $H_{k}(p):=\sum_{h=0}^{p-1} V_{2}\left(y_{k}(h)\right) \lambda^{p-1-h}$ it follows from (18) that

$$
\begin{aligned}
H_{k+1}(p) & \leq \lambda H_{k}(p)+\lambda^{p} V_{1}\left(x_{k+1}(0)\right) . \\
& -V_{1}\left(x_{k+1}(p)\right) .
\end{aligned}
$$


Solving the inequality (19) gives

$$
\begin{aligned}
H_{k}(p) \leq \lambda^{k} H_{0}(p) & +\sum_{n=0}^{k-1} \lambda^{k-1-n}\left(\lambda^{p} V_{1}\left(x_{n+1}(0)\right)\right. \\
& -V_{1}\left(x_{n+1}(p)\right)
\end{aligned}
$$

or

$$
\begin{array}{r}
\sum_{n=0}^{k-1} \lambda^{k-1-n} V_{1}\left(x_{n+1}(p)\right)+\sum_{h=0}^{p-1} \lambda^{p-1-h} V_{2}\left(y_{k}(h)\right) \\
\leq \lambda^{p} \sum_{n=0}^{k-1} \lambda^{k-1-n} V_{1}\left(x_{n+1}(0)\right)+\lambda^{k} \sum_{h=0}^{p-1} \lambda^{p-1-h} V_{2}\left(y_{0}(h)\right) .
\end{array}
$$

This last inequality is equivalent to

$$
\begin{array}{r}
\lambda^{-(p-1)} \sum_{n=0}^{k-1} \lambda^{-n} V_{1}\left(x_{n+1}(p)\right)+\lambda^{-(k-1)} \sum_{h=0}^{p-1} \lambda^{-h} V_{2}\left(y_{k}(h)\right) \\
\leq \lambda^{-(k-1)} \sum_{n=0}^{k-1} \lambda^{k-1-n} V_{1}\left(x_{n+1}(0)\right) \\
+\lambda^{-(p-1)} \sum_{h=0}^{p-1} \lambda^{p-1-h} V_{2}\left(y_{0}(h)\right)
\end{array}
$$

and evaluating of the right-hand side of (22) and using (3) and (16) gives

$$
\begin{aligned}
\lambda^{-(k-1)} \sum_{n=0}^{k-1} \lambda^{k-1-n} V_{1}( & \left.x_{n+1}(0)\right) \\
& +\lambda^{-(p-1)} \sum_{h=0}^{p-1} \lambda^{p-1-h} V_{2}\left(y_{0}(h)\right) \\
\leq & c_{2} \kappa_{d} \sum_{n=0}^{k-1} \lambda^{-n} \lambda_{d}^{n+1}+c_{2} M_{f} \sum_{h=0}^{T} \lambda^{-h} \\
\leq & c_{2} \kappa_{d} \sum_{n=0}^{\infty} \lambda^{-n} \lambda^{n} \lambda^{n}+c_{2} M_{f} \sum_{h=0}^{T} \lambda^{-h} \\
& =\frac{c_{2} \kappa_{d}}{1-\lambda}+\frac{c_{2} M_{f}\left(\lambda^{-T}-1\right)}{\lambda^{-1}-1}=C
\end{aligned}
$$


for all $k$ and $p$. Also it follows immediately from the left-hand side of (22) that

$$
\begin{aligned}
C \geq \lambda^{-(k-1)} \sum_{n=0}^{k-1} \lambda^{k-1-n} & V_{1}\left(x_{n+1}(0)\right) \\
& +\lambda^{-(p-1)} \sum_{h=0}^{p-1} \lambda^{p-1-h} V_{2}\left(y_{0}(p)\right) \\
& \geq c_{1} \lambda^{-(k-1)} \lambda^{-(p-1)}\left|x_{k}(p)\right|^{2}
\end{aligned}
$$

for all $k$ and $p$. Moreover

$$
\begin{aligned}
C \geq \lambda^{-(k-1)} \sum_{n=0}^{k-1} \lambda^{k-1-n} & V_{1}\left(x_{n+1}(0)\right) \\
& \\
+\lambda^{-(p-1)} \sum_{h=0}^{p-1} \lambda^{p-1-h} V_{2}\left(y_{0}(h)\right) & \quad \geq c_{1} \lambda^{-(k-1)} \lambda^{-(p-1)}\left|y_{k}(p-1)\right|^{2}
\end{aligned}
$$

for all $k$ and $p$. Finally, since $C<\infty$ it follows from (22)-(25) that (5) holds with $\kappa=\frac{C}{c_{1} \lambda^{2}}$ and the proof is complete.

Note that the rate of convergence $\lambda$ in this result does not depend on the pass length. The storage function (6) can be considered as a vector Lyapunov function for the controlled process formed by applying (12) to (1) that guarantees exponential stability.

\subsection{An example application}

In many industrial processes, the dynamics can be adequately approximated as linear for analysis and control law design but in implementation an actuator (or a sensor) becomes nonlinear due, for example, to saturation or hysteresis. One possible model for the dynamics in such a case is

$$
\begin{aligned}
x_{k+1}(p+1) & =A_{11} x_{k+1}(p)+A_{12} y_{k}(p)+\phi_{1}\left(x_{k+1}(p), y_{k}(p)\right) u_{k+1}(p), \\
y_{k+1}(p) & =A_{21} x_{k+1}(p)+A_{22} y_{k}(p)+\phi_{2}\left(x_{k+1}(p), y_{k}(p)\right) u_{k+1}(p),
\end{aligned}
$$

where $\phi_{1}$ and $\phi_{2}$ are nonlinear vector functions of $x$ and $y$ and in the simplest case $\phi_{1}$ and $\phi_{2}$ are constant vectors. Let

$$
\phi\left(x_{k+1}(p), y_{k}(p)\right)=\left[\phi_{1}^{T}\left(x_{k+1}(p), y_{k}(t)\right) \phi_{2}^{T}\left(x_{k+1}(p), y_{k}(p)\right)\right]^{T} .
$$


Also consider a square matrix, say $H$, and let $H \succ 0$ (respectively $H \prec 0$ ) denote the symmetric positive definite (respectively negative definite) property and choose a storage function in the form of (6) as

$$
\begin{aligned}
V_{1}\left(x_{k+1}(p)\right. & =x_{k+1}^{T}(p) P_{1} x_{k+1}(p) \\
V_{2}\left(y_{k}(p)\right) & =y_{k}^{T}(p) P_{2} y_{k}(p)
\end{aligned}
$$

where $P_{1} \succ 0$ and $P_{2} \succ 0$, satisfy the Lyapunov inequality

$$
\bar{A}^{T} P \bar{A}-P+Q \prec 0,
$$

with $\bar{A}=\left[\begin{array}{cc}A_{11} & A_{12} \\ A_{21} & A_{22}\end{array}\right], P=\left[\begin{array}{cc}P_{1} & 0 \\ 0 & P_{2}\end{array}\right], Q \succ 0$.

Computing the divergence of the vector function (6) gives

$$
\begin{array}{r}
\mathcal{D}_{d} V\left(x_{k+1}(p), y_{k}(p)\right)=\left[x_{k+1}^{T}(p), y_{k}^{T}(p)\right]\left(\bar{A}^{T} P \bar{A}\right. \\
-P)\left[x_{k+1}^{T}(p), y_{k}^{T}(p)\right]^{T}+\left[2\left[x_{k+1}^{T}(p), y_{k}^{T}(p)\right]^{T} \bar{A}^{T} P \phi\left(x_{k+1}(p), y_{k}(p)\right)\right. \\
+\phi^{T}\left(x_{k+1}(p), y_{k}(p)\right) P \phi\left(x_{k+1}(p), y_{k}(p)\right] u_{k+1}(p) .
\end{array}
$$

Define an auxiliary output vector for (26) as

$$
\begin{gathered}
z_{k+1}(p)=2 \phi^{T}\left(x_{k+1}(p), y_{k}(p)\right) P \bar{A}\left[x_{k+1}^{T}(p) y_{k}^{T}(p)\right]^{T} \\
+\phi^{T}\left(x_{k+1}(p), y_{k}(p)\right) P \phi\left(x_{k+1}(p), y_{k}(p)\right) u_{k+1}(p)
\end{gathered}
$$

and using (28) and (29) gives

$$
\begin{array}{r}
\mathcal{D}_{d} V\left(x_{k+1}(p), y_{k}(p)\right)=\left[x_{k+1}^{T}(p), y_{k}^{T}(p)\right]\left(\bar{A}^{T} P \bar{A}\right. \\
-P)\left[x_{k+1}^{T}(p), y_{k}^{T}(p)\right]^{T}+z_{k+1}^{T}(p) u_{k+1}(p) \\
\leq z_{k+1}^{T}(p) u_{k+1}(p)-\left[x_{k+1}^{T}(p), y_{k}^{T}(p)\right] Q\left[x_{k+1}^{T}(p), y_{k}^{T}(p)\right]^{T} .
\end{array}
$$

Hence it follows from (30) that a process described by (26) and (29) is exponentially $G$-passive with $G=I$, where $I$ denotes the identity matrix of compatible dimensions. Then using Theorem 1 the control law

$$
\begin{aligned}
u_{k+1}(p)=-[I & \left.+\phi^{T}\left(x_{k+1}(p), y_{k}(p)\right) P \phi\left(x_{k+1}(p), y_{k}(p)\right)\right]^{-1} \\
& \times 2 \phi^{T}\left(x_{k+1}(p), y_{k}(p)\right) P \bar{A}\left[x_{k+1}^{T}(p), y_{k}^{T}(p)\right]^{T},
\end{aligned}
$$

applied to (26) results in a controlled process that is exponentially stable. 


\section{Passivity and stabilization of differential repetitive processes}

Consider differential nonlinear repetitive processes with pass length $T<$ $\infty$ described over $0 \leq t \leq T$ by the state-space model

$$
\begin{aligned}
& \dot{x}_{k+1}(t)=f_{1}\left(x_{k+1}(t), y_{k}(t), u_{k+1}(t)\right), \\
& y_{k+1}(t)=f_{2}\left(x_{k+1}(t), y_{k}(t), u_{k+1}(t)\right),
\end{aligned}
$$

where on pass $k, x_{k}(t) \in \mathbb{R}^{n_{x}}$ is the state vector, $y_{k}(t) \in \mathbb{R}^{n_{y}}$ is the pass profile vector, $u_{k}(t) \in \mathbb{R}^{n_{u}}$ is the input vector; $f_{1}, f_{2}$ and $g$ are nonlinear functions such that $f_{1}(0,0,0)=0, f_{2}(0,0,0)=0$ and hence an equilibrium at zero.

The boundary conditions, i.e, the pass state initial vector sequence and the initial pass profile are assumed to be known and to satisfy the differential equivalent of the conditions given in (3) for discrete processes. Hence they are of the form

$$
\begin{aligned}
x_{k+1}(0) & =d_{k+1}, k \geq 0,\left|d_{k+1}\right|^{2} \leq \kappa_{d} \lambda_{d}^{k+1}, k \geq 0, \\
y_{0}(t) & =f(t),|f(t)|^{2} \leq M_{f}, 0 \leq t \leq T,
\end{aligned}
$$

where the entries in $d_{k+1} \in \mathbb{R}^{n_{x}}$ are known constants, the entries in $f(t) \in \mathbb{R}^{n_{y}}$ are known functions of $t$ and $\kappa_{d}>0,0<\lambda_{d}<1$. This model is the differential counterpart of the model considered in the Section 2.

Remark 1. In this and the next section, several assumed properties of the vector storage function will be invoked that are the natural counterparts for the discrete processes considered previously in this paper. From this point onwards, references to such properties must be interpreted in the differential repetitive process sense.

Introduce, in an analogous manner to the discrete case in Section 2, the auxiliary vector $z_{k}(t) \in \mathbb{R}^{n_{z}}$ given by

$$
z_{k+1}(t)=g\left(x_{k+1}(t), y_{k}(t), u_{k+1}(t)\right),
$$

where $g$ is a nonlinear function such that $g(0,0,0)=0$. Suppose also that on applying the control law

$$
u_{k+1}(t)=\psi\left(x_{k+1}(t), y_{k}(t)\right)
$$


to (32) the function $f_{1}$ satisfies the following Lipschitz condition with respect to variables $x$ and $y$ :

$$
\begin{aligned}
& \left|f_{1}\left(x^{\prime}, y^{\prime}, \psi\right)-f_{i}\left(x^{\prime \prime}, y^{\prime \prime}, \psi\right)\right| \leq L\left(\left|x^{\prime}-x^{\prime \prime}\right|+\left|y^{\prime}-y^{\prime \prime}\right|\right), \\
& \quad x^{\prime}, x^{\prime \prime} \in \mathbb{R}^{n_{x}}, y_{2}^{\prime}, y_{2}^{\prime \prime} \in \mathbb{R}^{n_{y}}, \psi \in \mathbb{R}^{n_{u}} .
\end{aligned}
$$

Exponential stability for the resulting controlled process is defined as follows.

Definition 3. Consider the differential nonlinear repetitive process resulting from the application of a control law of the form (35) to a process described by (32) and (33). Then the resulting controlled process is said to be exponentially stable if there exist real numbers $\kappa>0, \lambda>0$ and $0<\zeta<1$ such that

$$
\left|x_{k}(t)\right|^{2}+\left|y_{k}(t)\right|^{2} \leq \kappa \exp (-\lambda t) \zeta^{k} .
$$

To construct a control law of the form (35) that ensures exponential stability of the controlled process, the analysis that follows is based on the extension of passivity to differential nonlinear repetitive processes that also makes use of a vector storage function of the form (6). Also the divergence operator of this function along the trajectories of (32) is defined as

$$
\mathcal{D}_{c} V\left(x_{k+1}(t), y_{k}(t)\right)=\frac{d V_{1}\left(x_{k+1}(t)\right)}{d t}+\Delta_{k} V_{2}\left(y_{k}(t)\right)
$$

where $\Delta_{k} V_{2}\left(y_{k}(t)\right)=V_{2}\left(y_{k+1}(t)\right)-V_{2}\left(y_{k}(t)\right)$.

Definition 4. A differential nonlinear repetitive process described by (32) and (33) is said to be exponentially $G$ passive if there exists a vector function (6) and positive scalars $c_{1}, c_{2}$ and $c_{3}$ such that the conditions (9), (10) and

$$
\begin{aligned}
\mathcal{D}_{c} V\left(x_{k+1}(t), y_{k}(t)\right) & \leq z_{k+1}^{T}(t) G u_{k+1}(t) \\
& -c_{3}\left(\left|x_{k+1}(t)\right|^{2}+\left|y_{k}(t)\right|^{2}\right)
\end{aligned}
$$

hold, where $G$ is a constant matrix of compatible dimension and $z$ is given by (34).

Theorem 2. Suppose that a differential nonlinear repetitive process described by (32) and (33) is $G$ passive and there exists a positive scalar $c_{4}$ such that

$$
\left|\frac{\partial V_{1}(x)}{\partial x}\right| \leq c_{4}|x| .
$$


Suppose also that i) there exists a function $\varphi(z)$ such that $\varphi(0)=0$ and $z^{T} G \varphi(z)>0$ if $z \neq 0$ and ii) on application of a control law of the form (35) the function $f_{1}$ satisfies the Lipschitz conditions given in (36). Then the controlled process is exponentially stable.

Proof. It follows from (4) that there exists $\bar{c}_{3}<c_{3}$ such that $\lambda_{d}^{\frac{1}{2}}<\zeta:=$ $1-\frac{\bar{c}_{3}}{c_{2}}<1$ and using (12) gives

$$
\begin{array}{r}
\mathcal{D}_{c} V\left(x_{k+1}(t), y_{k}(t)\right) \leq-z_{k+1}^{T}(t) G \varphi\left(z_{k+1}(t)\right) \\
-\bar{c}_{3}\left(\left|x_{k+1}(t)\right|^{2}+\left|y_{k}(t)\right|^{2}\right) \leq-\bar{c}_{3}\left(\left|x_{k+1}(t)\right|^{2}+\left|y_{k}(t)\right|^{2}\right) .
\end{array}
$$

Also it follows from (4) and (41) that

$$
\begin{array}{r}
\frac{d V_{1}\left(x_{k+1}(t)\right)}{d t}+\lambda V_{1}\left(x_{k+1}(t)\right) \\
+V_{2}\left(y_{k+1}(t)\right)-\zeta V_{2}\left(y_{k}(t)\right) \leq 0,
\end{array}
$$

where $\lambda \in(0,1)$. Solving the inequality (42) with respect to $V_{1}\left(x_{k+1}(t)\right)$ gives

$$
\begin{array}{r}
V_{1}\left(x_{k+1}(t)\right) \leq V_{1}\left(x_{k+1}(0)\right) \mathrm{e}^{-\lambda t} \\
-\int_{0}^{t} \mathrm{e}^{-\lambda(t-s)}\left[V_{2}\left(y_{k+1}(s)\right)-\zeta V_{2}\left(y_{k}(s)\right)\right] d s .
\end{array}
$$

Introducing

$$
\begin{aligned}
W_{k+1}(t) & :=V_{1}\left(x_{k+1}(0)\right) \mathrm{e}^{-\lambda t}-V_{1}\left(x_{k+1}(t)\right), \\
H_{k}(t) & :=\int_{0}^{t} \mathrm{e}^{-\lambda(t-s)} V_{2}\left(y_{k}(s)\right) d s .
\end{aligned}
$$

enables (43) to be rewritten as

$$
H_{k+1}(t) \leq \zeta H_{k}(t)+W_{k+1}(t) .
$$

Solving the inequality (44) gives

$$
H_{n}(t) \leq \zeta^{n} H_{0}(t)+\sum_{k=1}^{n} W_{k}(t) \zeta^{n-k}
$$


or

$$
\begin{aligned}
& \sum_{k=1}^{n} V_{1}\left(x_{k}(t)\right) \zeta^{n-k}+\int_{0}^{t} \mathrm{e}^{-\lambda(t-s)} V_{2}\left(y_{n}(s)\right) d s \\
& \leq \mathrm{e}^{-\lambda t} \sum_{k=1}^{n} V_{1}\left(x_{k}(0)\right) \zeta^{n-k} \\
&+\zeta^{n} \int_{0}^{t} \mathrm{e}^{-\lambda(t-s)} V_{2}\left(y_{0}(s)\right) d s .
\end{aligned}
$$

The last inequality is equivalent to

$$
\begin{aligned}
& \mathrm{e}^{\lambda t} \sum_{k=1}^{n} V_{1}\left(x_{k}(t)\right) \zeta^{-k}+\zeta^{-n} \int_{0}^{t} \mathrm{e}^{\lambda s} V_{2}\left(y_{n}(s)\right) d s \\
& \leq \zeta^{-n} \sum_{k=1}^{n} V_{1}\left(x_{k}(0)\right) \zeta^{n-k} \\
&+\mathrm{e}^{\lambda t} \int_{0}^{t} \mathrm{e}^{-\lambda(t-s)} V_{2}\left(y_{0}(s)\right) d s .
\end{aligned}
$$

Evaluation of the right hand side of (46) (recalling the assumption on the boundary conditions) gives

$$
\begin{aligned}
\zeta^{-n} \sum_{k=1}^{n} V_{1}\left(x_{k}(0)\right) \zeta^{n-k} & +\mathrm{e}^{\lambda t} \int_{0}^{t} \mathrm{e}^{-\lambda(t-s)} V_{2}\left(y_{0}(s)\right) d s \\
\leq & \sum_{k=1}^{n} c_{2} \kappa_{d} \lambda_{d}^{k} \zeta^{-k}+\int_{0}^{t} c_{2} M_{f} \mathrm{e}^{\lambda s} d s \\
\leq & \sum_{k=1}^{n} c_{2} \kappa_{d}\left(\lambda_{d}^{\frac{1}{2}}\right)^{k}\left(\lambda_{d}^{\frac{1}{2}}\right)^{k} \zeta^{-k}+\frac{c_{2} M_{f}\left(\mathrm{e}^{\lambda t}-1\right)}{\lambda} \\
\leq & \sum_{k=1}^{n} c_{2} \kappa_{d} \zeta^{k} \zeta^{k} \zeta^{-k}+\frac{c_{2} M_{f}\left(\mathrm{e}^{\lambda T}-1\right)}{\lambda} \\
\leq \sum_{k=1}^{\infty} c_{2} \kappa_{d} \zeta^{k}+\frac{c_{2} M_{f}\left(\mathrm{e}^{\lambda T}-1\right)}{\lambda} & =\frac{c_{2} \kappa_{d}}{1-\zeta}+\frac{c_{2} M_{f}\left(\mathrm{e}^{\lambda T}-1\right)}{\lambda}=C .
\end{aligned}
$$


and it follows from (46) and (47) that

$$
c_{1}\left|x_{n}(t)\right|^{2} \zeta^{-n} \mathrm{e}^{\lambda t} \leq C \text { for all } t \in[0, \infty], n=0,1, \ldots
$$

Evaluating $\frac{d V_{1}(x)}{d t}$ and using the Lipschitz conditions (36) and (40) gives

$$
\begin{array}{r}
\frac{d V_{1}\left(x_{k+1}(t)\right)}{d t}=\frac{\partial V_{1}\left(x_{k+1}(t)\right)}{\partial x_{k+1}(t)} f_{1}\left(x_{k+1}(t), y_{k}(t),-\varphi\left(z_{k+1}(t)\right)\right) \geq \\
\geq-\left|\frac{\partial V_{1}\left(x_{k+1}(t)\right)}{\partial x_{k+1}(t)}\right|\left|f_{1}\left(x_{k+1}(t), y_{k}(t),-\varphi\left(z_{k+1}(t)\right)\right)\right| \geq \\
\geq-c_{4} L\left(\left|x_{k+1}(t)\right|+\varepsilon\left|y_{k}(t)\right|\right)\left(\left|x_{k+1}(t)\right|+\left|y_{k}(t)\right|\right) \geq \\
\geq-\alpha V_{1}\left(x_{k+1}(t)-\beta \varepsilon V_{2}\left(y_{k}(t)\right),\right.
\end{array}
$$

where $\alpha=\frac{c_{4} L(\varepsilon+1)^{2}}{c_{1} \varepsilon}, \beta=\frac{c_{4} L}{c_{1}}$, and $\varepsilon$ is arbitrary positive scalar. It follows from (42) and (49) that

$$
V_{2}\left(y_{k+1}(t)\right)-z_{0} V_{2}\left(y_{k}(t)\right) \leq \alpha V_{1}\left(x_{k+1}(t)\right)
$$

where $z_{0}=\zeta+\beta \varepsilon$. Choosing $\varepsilon$ small enough such that $0<z_{0}<1$, solving (50) and using (48) gives

$$
V_{2}\left(y_{n}(t)\right) \leq z_{0}^{n} V_{2}\left(y_{0}(t)\right)+\frac{\alpha c_{2}}{c_{1}} \sum_{k=1}^{n} z_{0}^{n-k} \zeta^{k} \mathrm{e}^{-\lambda t}
$$

This last inequality and (33) establishes that the function $V_{2}\left(y_{n}(t)\right) \zeta_{0}^{-n} \mathrm{e}^{\lambda t}$ with $\zeta_{0}>z_{0}$ is bounded for all $t \in[0, \infty], n=0,1, \ldots$ Moreover, using (10) gives

$$
\left|y_{n}(t)\right|^{2} \leq \bar{C} \zeta_{0}^{n} \mathrm{e}^{-\lambda t}
$$

where $\bar{C}$ is a positive constant. It follows immediately from (48) and (51) that (5) holds and the proof is complete.

Note that as in case of discrete processes both $\zeta$ and $\lambda$, defining the rate of convergence, do not depend on the pass length. Moreover, the example application for the discrete process results given in Section 2.3 extend in a natural manner to differential dynamics and hence the details are omitted. 


\section{4. $H_{\infty}$ stabilization and disturbance attenuation}

In applications, repetitive processes may also be subject to disturbances and this section extends the stabilizing control law design to also include disturbance rejection. Again, the results derived hold for discrete or differential dynamics and hence this section only considers the latter case.

Consider a differential nonlinear repetitive processes in the presence of disturbances described over $0 \leq t \leq T<\infty$ by the state-space model

$$
\begin{aligned}
& \dot{x}_{k+1}(t)=f_{1}\left(x_{k+1}(t), y_{k}(t), u_{k+1}(t), w_{k}(t)\right), \\
& y_{k+1}(t)=f_{2}\left(x_{k+1}(t), y_{k}(t), u_{k+1}(t), w_{k}(t)\right),
\end{aligned}
$$

where $x_{k}(t) \in \mathbb{R}^{n_{x}}, y_{k}(t) \in \mathbb{R}^{n_{y}}$ and $u_{k}(t) \in \mathbb{R}^{n_{u}}$ are as in (32) and $w_{k}(t) \in$ $\mathbb{R}^{n_{w}}$ is a disturbance vector, $f_{1}$ and $f_{2}$ are nonlinear functions such that $f_{1}(0,0,0,0)=0, f_{2}(0,0,0,0)=0$ (and hence an equilibrium at zero) and both $x$ and $y$ are continuous functions of $t$. The boundary conditions are given by (33). In this case the dissipativity property is studied with respect to the disturbance input $w$ with the pass profile vector $y$ treated as the output. Assume also that $w_{k}(t) \in L_{2}([0, \infty),[0, \infty))$ and define

$$
\|w\|_{2}=\sqrt{\sum_{k=0}^{\infty} \int_{0}^{\infty}\left|w_{k}(t)\right|^{2} d t}<\infty .
$$

Definition 5. A differential nonlinear repetitive process described by (52) and $u_{k+1}(t)=\psi\left(x_{k+1}(t), y_{k}(t)\right), \psi(0,0)=0$ is said to be exponentially stable with prescribed $H_{\infty}$ disturbance attenuation level $\gamma$ if it is exponentially stable for $w_{k}(t)=0$ and for $w_{k}(t) \in L_{2}([0, \infty),[0, \infty))$ and zero boundary conditions

$$
\|y\|_{2}<\gamma\|w\|_{2}
$$

Consider a process described by $(52)$ and $w_{k}(t) \in L_{2}([0, \infty),[0, \infty))$. Then the following result can be established.

Theorem 3. Assume that for some $u=\psi(x, y), \psi(0,0)=0$ function $f_{1}$ satisfies Lipschitz condition (36) and the inequality

$$
\begin{array}{r}
\mathcal{D}_{c} V\left(x_{k+1}(t), y_{k}(t)\right)+\left|x_{k+1}(t)\right|^{2}+\left|y_{k}(t)\right|^{2} \\
-\gamma^{2}\left|w_{k}(t)\right|^{2} \leq 0
\end{array}
$$


has a solution $V(x, y)$ satisfying (9), (10) and (40). Then the controlled process resulting from applying $u_{k+1}(t)=\psi\left(x_{k+1}(t), y_{k}(t)\right)$ to (52) is exponentially stable with prescribed $H_{\infty}$ disturbance attenuation level $\gamma$.

Proof. Let the pair $(V(x, y), \varphi(x, y))$ be a solution of $(54)$. If $w_{k}(t)=0$, it follows from (54) that

$$
\mathcal{D}_{c} V\left(x_{k+1}(t), y_{k}(t)\right) \leq-\left(\left|x_{\varphi, k+1}(t)\right|^{2}+\left|y_{\varphi, k}(t)\right|^{2}\right)
$$

and by Theorem 2 the controlled process is exponentially stable. Now consider $w_{k}(t) \in L_{2}([0, \infty),[0, \infty))$ and then it follows from (55) that

$$
\begin{array}{r}
\frac{d V_{1}\left(x_{k+1}(t)\right)}{d t}+V_{2}\left(y_{k+1}(t)\right)-V_{2}\left(y_{k}(t)\right) \\
\leq-\left(\left|x_{k+1}(t)\right|^{2}+\left|y_{\varphi, k}(t)\right|^{2}-\gamma^{2}\left|w_{k}(t)\right|^{2}\right) \\
\leq-\left(\left|y_{k}(t)\right|^{2}-\gamma^{2}\left|w_{k}(t)\right|^{2}\right) .
\end{array}
$$

Integrating and summing both the sides of (56) and rearranging the summands gives

$$
\begin{array}{r}
\sum_{k=0}^{n} \int_{0}^{t}\left|y_{k}(s)\right|^{2} d s \leq \sum_{k=0}^{n} \int_{0}^{t} \gamma^{2}\left|w_{k}(s)\right|^{2} d s \\
-\sum_{k=0}^{n} V_{1}\left(x_{k+1}(t)\right)-\int_{0}^{t} V_{2}\left(y_{k}(t)\right) \\
\leq \sum_{k=0}^{n} \int_{0}^{t} \gamma^{2}\left|w_{k}(s)\right|^{2} d s .
\end{array}
$$

It follows on letting $n, t \rightarrow \infty$ in (57) that (53) holds and the proof is complete.

\section{Conclusions}

This paper has developed the first results on the use of passivity in the stability analysis and control law design for nonlinear repetitive processes. Repetitive processes are a distinct class of $2 \mathrm{D}$ systems that have physical applications and, in particular, for linear dynamic model based ILC laws defined in this setting have been experimentally verified. Overall, these results provide a basis for onward research with the long-term aim of providing a mature setting for control law design. To further enhance the subject area, further research should also consider the case when the state initial vector or each pass is an explicit function of the previous pass profile vector. 


\section{References}

[1] E. Rogers, K. Gałkowski, and D.H. Owens. Control Systems Theory and Applications for Linear Repetitive Processes. Lecture Notes in Control and Information Sciences, Vol. 349. Springer-Verlag, Berlin, 2007.

[2] L. Hladowski, K. Galkowski, Z. Cai, E. Rogers, C.T. Freeman, and P.L. Lewin. Experimentally supported 2D systems based iterative learning control law design for error convergence and performance. Control Engineering Practice, 18:339- 348, 2010.

[3] T. P. Azevedo-Perdicoulis and G. Jank. Disturbance attenuation of linear quadratic OL-Nash games on repetitive proceses with smoothing on the gas dynamics. Multidimensional Systems and Signal Processing, 23:135-153, 2012.

[4] P. Pakshin, K. Gałkowski, and E. Rogers. Stability and stabilization of systems modeled by 2D nonlinear stochastic Roesser models. In Proc. 7th Int. Workshop on Multidimensional (nD) systems, pages 1-6. Poitiers, France, September 5-7, 2011.

[5] Nima Yeganefar, Nader Yeganefar, M. Ghamgui, and E. Moulay. Lyapunov theory for 2-D nonlinear Roesser models: Application to asymptotic and exponential stability. IEEE Transactions on Automatic Control, 58:1299-1304, 2013.

[6] P. M. Sammons, D. A. Bristow, and R. G. Landers. Height dependent laser metal deposition process modeling. Journal of Manufacturing Science and Engineering, (5):054501-054501-8, 2013.

[7] S. Hara, Y. Yamamoto, T. Omata, and M. Nakano. Repetitive control system: a new type servo system for periodic exogenous signals. IEEE Transactions on Automatic Control, 33(7):659-668, 1988.

[8] P. D. Roberts. Two-dimensional analysis of an iterative nonlinear optimal control algorithm. IEEE Transactions on Circuits and Systems I: Fundamental Theory and Applications, 49(6):872-878, 2002.

[9] J.C.Willems. Dissipative dynamical systems part I: General theory. Archive for Rational Mechanics and Analysis, 45:321-351, 1972. 
[10] C.I. Byrnes, A. Isidori, and J.C. Willems. Passivity, feedback equivalence and the global stabilization of minimun phase nonlinear systems. IEEE Transactions on Automatic Control, 36:1228 - 1240, 1991.

[11] W. Haddad, Q.Hui, V. Chellabona, and S. Nersesov. Vector dissipativity theory for discrete-time large-scale nonlinear dynamical systems. Advances in Difference Equations, 1:37 - 66, 2001.

[12] W. Haddad, V. Chellabona, and S. Nersesov. Vector dissipativity theory and stability of feedback interconnections for large-scale non-linear dynamical systems. International Journal of Control, 77:907-919, 2004.

[13] J. Emelianova, P. Pakshin, K. Gałkowski, and E. Rogers. Vector Lyapunov function based stability of a class of applications relevant 2D nonlinear systems. In Proceedings of the 19th IFAC World Congress, pages 8247-8252. Cape Town South Africa, August 24-29, 2014.

[14] J. Emelianova, P. Pakshin, K. Gałkowski, and E. Rogers. Stability of nonlinear discrete repetitive processes with Markovian switching. Systems \&3 Control Letters, 75:108-116, 2015.

[15] A.L. Fradkov and D.J. Hill. Exponential feedback passivity and stabilizability of nonlinear systems. Automatica, 34:697-703, 1998.

[16] H.K. Khalil. Nonlinear Systems. Third Edition. Prentice Hall, New Jersey, 2002. 